\title{
Diacronie
}

Studi di Storia Contemporanea

$\mathrm{N}^{\circ} 11,3$ | 2012

La satira fa storia. Eventi, pratiche, linguaggi

\section{Khaled Fouad Allam, L'Islam spiegato ai leghisti}

\section{Luca Zuccolo}

\section{(2) OpenEdition}

\section{Journals}

\section{Edizione digitale}

URL: http://journals.openedition.org/diacronie/2725

DOI: $10.4000 /$ diacronie. 2725

ISSN: 2038-0925

\section{Editore}

Association culturelle Diacronie

Notizia bibliografica digitale

Luca Zuccolo, « Khaled Fouad Allam, L'Islam spiegato ai leghisti », Diacronie [Online], N 11, 3 | 2012,

documento 12, Messo online il 29 octobre 2012, consultato il 24 septembre 2020. URL : http://

journals.openedition.org/diacronie/2725; DOI : https://doi.org/10.4000/diacronie.2725 


\section{Diacronie}

N. 11 | 3|2012 La satira fa storia. Eventi, pratiche, linguaggi

12/

\section{RECENSIONE:}

\section{Khaled Fouad ALLAM, L'Islam spiegato ai leghisti, Milano, Piemme, 2011, 182 pp.}

a cura di Luca ZUCCOLO *

Ponendosi alla "frontiera" tra «ciò che stiamo lasciando e l'enigma del domani»"1 Allam, autore tra l'altro di alcuni bestsellers quali L'Islam globale e La solitudine dell'Occidente $^{3}$, attraverso il libro, L'Islam spiegato ai leghisti, propone un nuovo approccio ad una delle più stringenti questioni del XXI secolo: il rapporto tra l'Islam e l'Occidente. A metà tra racconto personale e analisi sociologica il saggio di Allam, sociologo e politico di origine algerina, cittadino italiano dal 1993 dopo una vita passata tra Marocco, Algeria e Francia, pone nuovi interrogativi sul nostro futuro e sul rapporto dell'Italia e dell'Europa con il mondo islamico.

Il saggio si articola in dodici capitoli molto agili e ben scritti in cui i fatti dell'attualità si integrano e si intrecciano perfettamente all'analisi storico-sociologica e alla spiegazione delle principali teorie e problematiche relative al rapporto tra Italia, Europa e mondo islamico.

Il primo capitolo, che funge da introduzione, propone un quadro della realtà sociale italiana contemporanea, sempre più multietnica e globalizzata, e inizia a porre la questione centrale del libro ovvero il rapporto con la diversità e la visione della questione da parte della Lega Nord.

Il secondo capitolo apre la questione principale del saggio, ovvero la dialettica e il discorso politico della Lega. Allam propone un'analisi del fenomeno leghista e di come, paradossalmente, la Lega e la sua retorica politica siano scarsamente studiate e indagate in modo approfondito. Nondimeno, l'autore mette in luce due dati a mio avviso fondamentali per comprendere il fenomeno leghista e il suo rapporto con l'Islam. Da un lato, sottolinea come il discorso leghista proponga una dinamica molto

${ }^{1}$ ALLAM, Khaled Fouad, L'Islam spiegato ai leghisti, Milano, Piemme, 2011, p. 13.

${ }^{2}$ ALLAM, Khaled Fouad, L'Islam globale, Milano, Rizzoli, 2002.

3 ALLAM, Khaled Fouad, La solitudine dell'Occidente, Milano, Rizzoli, 2006. 
sfumata riguardo al razzismo e non presenti al suo interno alcun testo di riferimento chiaramente xenofobo, ma piuttosto proponga una dinamica politica "di pancia" che sfrutta le paure e gli stereotipi delle masse. Dall'altro lato, l'autore mette in luce come la Lega sia l'unico movimento politico che in Italia abbia posto il problema dell'immigrazione e delle relazioni con l'universo islamico.

Interessante a questo proposito è la citazione tratta dall'articolo di Giovanni De Luna del 1992 dal titolo «Strategie della paura» che sottolinea la peculiarità del discorso leghista:

È importante lo scenario conflittuale in cui avvenne allora e continua oggi l'“invenzione della tradizione”. Non bastava creare artificiosamente dal nulla un'identità e un'appartenenza regionale: occorreva caricarla di valenze antagonistiche, darle dei nemici contro cui indirizzarsi. È stato questo il capolavoro "soggettivo" di Umberto Bossi e dei suoi amici. Si è lombardi (o piemontesi, o veneti) essenzialmente contro Roma, contro i partiti "nazionali", contro lo Stato, contro i meridionali, contro gli extracomunitari, contro i drogati contro gli omosessuali, e giù, lungo un elenco infinito di nemici reali o potenziali. Fu all'interno di queste coordinate che la manipolazione della Lega scoprì il "razzismo" [...]. È certo però che il razzismo della Lega è essenzialmente il programma di un partito politico, un elemento politicamente intenzionale, che tende a farlo apparire "come se fosse un carattere tipico e normale di una certa comunità" per utilizzarlo consapevolmente nel discorso elettorale ${ }^{4}$.

Il Leitmotiv leghista quindi è una sostanziale stigmatizzazione dell'Islam, la quale si inserisce nel più ampio dibattito europeo sull'immigrazione e sull'integrazione, sviluppatosi essenzialmente attorno ai concetti politici del modello assimilazionista francese e del modello comunitarista britannico.

All'analisi di questi due modelli, frutto delle esperienze coloniali dei due paesi europei, segue un'analisi della mancata elaborazione di una teoria dell'immigrazione in Italia e delle motivazioni di questa mancanza. Ad essa si aggiunge un interessante spunto di dibattito sulle problematiche che devono affrontare le seconde e le terze generazioni di immigrati nel nostro paese, spesso emarginate dalla società in cui sono cresciute, che tendono a ricostruire un'identità comunitaria propria e una forte tendenza ad una re-islamizzazione poiché vedono nell'Islam un fondamentale elemento identitario. La problematica coinvolge non solo l'Italia ma tutta l'Europa e viene ripresa

4 DE LUNA, Giovanni, Strategie della Paura, in L'opera al rosso, vol. 2, Genova, Marietti, 1992, p. 178, cit. in ALLAM, Khaled Fouad, op.cit., p. 18. 
nel paragrafo conclusivo, che introduce il tema del rapporto tra fenomeni migratori e presenza islamica, ponendo al centro della questione proprio le seconde generazioni.

Il terzo capitolo, proseguendo su questa linea interpretativa, si pone il problema delle frontiere reali o immaginate che dagli anni '9o si fanno sempre più rigide nei confronti dell'Islam e dell'immigrazione islamica, identificando nell'immigrato un capro espiatorio, colpevole perché, in virtù del suo status di soggetto sospeso tra la patria di provenienza e lo Stato di accoglienza, si trova ad essere fuori da ogni sistema di valori riconosciuto. A questo riguardo, Allam propone una posizione intermedia tra le teorie sulla globalizzazione e le nuove spinte identitarie emerse in reazione dai fenomeni globalizzanti, cercando di dimostrare come l'Islam non sia solo un espressione radicale e fondamentalista.

Poste queste premesse, l'autore elabora nei capitoli 4 e 5 un altro tema decisivo nella relazione tra Europa e Islam: l'islamofobia europea.

In questi due capitoli analizza dettagliatamente i motivi principali che provocano, da un lato l'islamofobia popolare, dall'altro l'islamofobia "colta", che viene ritenuta molto più deleteria poiché impedisce una reale integrazione e non favorisce la comprensione della cultura islamica, contribuendo alla diffusione di stereotipi e falsi miti. «L’islamofobia “colta”» infatti, «rappresenta un cortocircuito della storia, perché vuole accreditare la tesi che l'islam sia stato e sia tutt'oggi una religione di conquista: così si costruisce la macchina della paura, si rafforzano gli stereotipi mantenendo e rafforzando un'immagine negativa dei musulmani. E non è un caso che nel discorso politico contemporaneo, le tesi islamofobiche penetrino diversi segmenti politici» 5 .

Per meglio approfondire questi fenomeni Allam si riferisce, presentandone gli estratti più significativi, a saggi di storici e sociologi che permettono una migliore comprensione delle paure europee e del nuovo linguaggio anti-islamico sviluppato da alcuni settori della società europea, spaventati da una possibile perdita dell'identità europea in favore di un'Eurabia islamizzata.

Questa parte del testo di Allam si inserisce nel più ampio dibattito sulle radici europee e, a mio parere, pone degli interessanti spunti di analisi e di dibattito su un tema troppo spesso strumentalizzato come quello dell'identità europea. Il sottotesto di Allam, secondo cui l'Islam sia in qualche modo co-artefice di un'identità europea che non può essere limitata ai soli aspetti cristiani della questione, mi pare molto utile per proporre finalmente una visione dell'Europa più inclusiva rispetto alle visioni esclusive proposte da più parti e da più partiti, non ultimo la Lega. Una visione che consenta una

5 Ibidem, p. 67. 
dinamica sociale molto più aperta e tollerante verso i musulmani, ma che contempli anche una coscienza del meticciato presente da secoli lungo le sponde del Mediterraneo e nelle terre d'Europa.

Il sesto capitolo, il più interessante e stimolante del saggio, invece, propone un'immagine dell'Islam presa da una diversa angolazione: la tradizione degli umanisti musulmani.

In queste pagine Allam, approfondendo una rappresentazione troppo spesso appiattita sulle paure e sugli stereotipi orientalisti, come dimostrato dall'islamofobia "colta", descrive e recupera alcuni esempi di umanesimo islamico dimostrando come l'Islam non sia costituito solo dalle realtà fondamentaliste e radicali. Il dato rilevante di questo capitolo non è solo quello di proporre una visione dell'Islam inedita, ma soprattutto di sottolineare la profondità intellettuale e culturale del mondo islamico, troppo spesso dimenticata da politici e intellettuali europei.

Nel capitolo seguente (capitolo 7) l'autore descrive con diversi e significativi esempi il difficile incontro e il rapporto con l'Occidente e la modernità da parte delle società musulmane. Interessante si dimostra il parallelo con le popolazioni slave e la cultura russa utilizzato per spiegare come al loro interno né l'Occidente, né l'Islam siano due monoliti. Il capitolo si chiude con la descrizione dell'incontro-scontro tra Islam e modernità durante il XIX e XX secolo.

Nell'ottavo capitolo viene ripreso il tema leghista analizzandone la formazione del consenso, politico e popolare, che si basa essenzialmente sulle emozioni legate ad una possibile perdita dell'identità italiana ed europea di fronte al pericolo islamico. Allam ricorda come i leghisti, oltre ad essere a conoscenza della questione islamica e delle problematiche che essa comporta, siano stati i primi in Italia a proporre un confronto e un approccio politico al riguardo. Nondimeno, Allam, prendendo ad esempio la Piccola guida alla cultura islamica ${ }^{6}$ proposta da Edouard Ballaman, dimostra come le conoscenze leghiste sull'Islam siano spesso superficiali e manchino totalmente della profondità critico-scientifica necessaria per operare una critica costruttiva al mondo islamico. Come ricorda Allam, infatti, l'Islam non è riducibile al solo Corano. Al contrario è opportuno e necessario indagare e spiegare quanto è acceduto e accade nelle società musulmane attraverso l'analisi di tradizioni composite e di contingenze storico politiche ben determinate che hanno influenzato in modo decisivo non solo le società in oggetto ma la stessa religione musulmana. Una migliore comprensione dei lavori e delle vite dei principali pensatori musulmani, come propone Allam stesso (capitolo 6) è

${ }^{6}$ BALLAMAN, Edouard, Piccola guida alla cultura islamica,

URL: < http://www.ballaman.com > [consultato il 21 aprile 2012]. 
imprescindibile per comprendere a fondo una cultura complessa e diversificata come quella islamica.

La necessità di approfondire le conoscenze sull'Islam e la sua cultura riemerge nel capitolo nono dove Allam tocca tre dei prinicipali nodi attorno a cui ruotano le paure europee: la donna, la democrazia e la libertà religiosa. Questi tre temi problematici vengono analizzati da Allam attraverso una disamina delle dinamiche storiche e dell'attualità che vengono intrecciate in un discorso ben strutturato in grado di chiarire alcuni dei punti più discussi nel dibattito europeo.

Il capitolo decimo, invece, descrive e pone in evidenza le questioni ancora aperte che l'Occidente ha nei confronti del mondo musulmano, con particolare attenzione al multiculturalismo, al problema dell'immigrazione e al più spinoso problema dell'adesione della Turchia all'Unione Europea. Tutte questioni che pongono nuove domande e mettono in crisi l'attuale definizione di identità europea e che, se resteranno irrisolte, porranno dei limiti decisivi allo sviluppo socio-politico dell'area euromediterranea.

Gli ultimi due capitoli, infine, presentano episodi personali accaduti all'autore, il quale descrive delle vicende di integrazione e convivenza pacifica che superano le bieche logiche politiche e propagandistiche dimostrando come l'integrazione sia un obiettivo raggiungibile e sia sostanzialmente la realtà più comune dell'area mediterranea, da sempre teatro e spazio di incontri e commistioni culturali, sociali, economiche e politiche. Un insieme di incontri e relazioni che hanno contribuito all'arricchimento di tutte le popolazioni mediterranee e in primis dell'Italia.

Il saggio di Allam, nel suo complesso, oscilla tra l'analisi storico-sociologica e l'attualità, trovando un ottimo e proficuo equilibrio tra questi due aspetti e districandosi egregiamente attraverso i temi più caldi emersi negli ultimi anni nel dibattito europeo. L'autore con uno stile chiaro e scorrevole riesce a spiegare dettagliatamente e criticamente tematiche spinose, permettendo anche al vasto pubblico, a cui è indirizzato il libro, di destreggiarsi fra temi complessi e ancora poco noti nonostante la cospicua propaganda a riguardo.

All'interno del saggio risultano molto interessanti gli estratti proposti all'inizio o nel corpo dei singoli capitoli, funzionali alla comprensione dei temi trattatati e al loro approfondimento. Essi si presentano come un ottimo punto di partenza per chiunque volesse accrescere la propria conoscenza sul mondo islamico e sulle problematiche sollevate da Allam. Nondimeno, il valore di questo testo si trova nelle domande e negli stimoli analitici che emergono da ogni singola pagina del testo e che consentono di 
aprire nuove prospettive analitiche e di indagine, a cui si unisce una proposta concreta per una prossima e migliore integrazione sociale sia in Italia che in Europa. 


\section{* L'autore}

Luca Zuccolo, dottore (PhD) in Storia Contemporanea del SUM di Napoli ha sviluppato una ricerca sulle dinamiche di formazione dell'identità ottomana e di un discorso di tipo patriottico durante il regno di Abdülhamid II (1880-1885) attraverso lo studio di fonti a stampa redatte in lingua francese. Già dottore magistrale in Storia d'Europa (Bologna 2008), si è occupato dello sviluppo della modernità durante l'ultimo secolo dell'Impero Ottomano, del confronto/scontro tra modernità e tradizione in un contesto cosmopolita e allo sviluppo dei movimenti sociali che hanno preparato l'avvento della società turca contemporanea.

URL: <www.studistorici.com/2010/12/01/luca-zuccolo >

\section{Per citare questo articolo:}

ZUCCOLO, Luca, «Recensione: Khaled Fouad ALLAM, L'Islam spiegato ai leghisti, Milano, Piemme, 2011, 182 pp.», Diacronie. Studi di Storia Contemporanea: La satira fa storia. Eventi, pratiche, linguaggi, 29/10/2012,

URL:< http://www.studistorici.com/2012/10/29/zuccolo2_numero_11/ >

\section{Diacronie Studi di Storia Contemporanea $\beta$ www.diacronie.it}

Risorsa digitale indipendente a carattere storiografico. Uscita trimestrale. redazione.diacronie@hotmail.it

Comitato di redazione: Marco Abram - Giampaolo Amodei - Jacopo Bassi - Luca Bufarale - Gianluca Canè - Alessandro Cattunar - Alice De Rensis - Barbara Galimberti - Deborah Paci - Fausto Pietrancosta - Matteo Tomasoni - Luca Zuccolo 